\title{
An Analytical Treatment of the Energy Spectrum of Hydrogen-Like Atoms Perturbed by a Generalized van der Waals Potential
}

\author{
M.E. Amin \\ Mathematics Department, Faculty of Science, Minia University, El-Minia, Egypt \\ M.A El-Aasser \\ Physics Department, Faculty of Science, Ain Shams University, Cairo, Egypt \\ (Received on 26 January, 2009)
}

\begin{abstract}
Hydrogen-like atoms are two-particle systems governed with spherically symmetric potentials, their nonrelativistic Schrödinger equations can be solved analytically yielding exact information about their spectra. Vigorous interest in the investigation of their behavior under the influence of external fields has been increased over the years. The main goal of the present work is to investigate the effect of a generalized van der Waals potential on the energy spectra of the hydrogen-like atoms. Our treatment is based on the Oscillator Representation Method (ORM). The energy spectrum is studied for various parameters over an atom-surface distance $50 \mathrm{~nm}-300 \mathrm{~nm}$ for different hydrogen-like atoms, like $\mathrm{H}, \mathrm{He}^{+}, \mathrm{Li}^{2+}, \mathrm{Be}^{3+}, \mathrm{B}^{4+}$, etc.
\end{abstract}

Keywords: Hydrogen-like atoms, Generalized van der Waals Forces, Oscillator Representation Method.

\section{INTRODUCTION}

It is well known that a hydrogen-like atom is composed of a single electron of charge - e moving around a positive nucleus of charge $+Z e$. It is one of few exactly solvable quantum mechanical problems, whose eigenvalues and eigenfunctions can be determined exactly. However, in the presence of external fields the atom shows a variety of nonlinear phenomena depending upon the nature of perturbations (see Series 1988). By using the method of images, the Hamiltonian of the hydrogen-like atom can be written as

$$
H=\frac{1}{2} P^{2}-\frac{Z}{r}-\frac{Z^{2}}{4 D}+\frac{Z}{\sqrt{\rho^{2}+(2 D+z)^{2}}}-\frac{1}{4(D+z)},
$$

where $(x ; y ; z)$ are the electron coordinates relative to the nucleus, $2 D$ is the distance between the nucleus and the image nucleus, $Z$ is the charge of the nucleus and $r$ is the distance between the electron of the atom and the nucleus. The cylindrical coordinates $\rho, \varphi$ and $z$ are related to the Cartesian coordinates by: $\mathrm{x}=\rho \cos \varphi, y=\rho \sin \varphi$ and $z=z$. The used units are Hartree atomic units in terms of which $h=e=m$ $=1$, where $e$ is the charge of the electron, $m$ is its mass, and $h$ is planck's constant. In the special case of $Z=1$, when the nucleus is a proton, the hydrogen-like atom is an ordinary hydrogen atom.

Assuming that the atom is at a distance from the surface much larger than the size of atom i.e. $D^{2}>>r^{2}$ and by using the binomial expansion, retaining only the quadratic terms, the generalized van der Waals Hamiltonian is obtained by

$$
\begin{aligned}
H= & \frac{1}{2} P^{2}-\frac{Z}{r}-\frac{(1-Z)^{2}}{4 D}+\frac{(1-Z) z}{4 D^{2}}-\frac{1}{16 D^{3}} \times \\
& \times\left[Z \rho^{2}+\beta^{2} z^{2}(2-Z)\right]
\end{aligned}
$$

Furthermore, the energy spectrum of the instantaneous van der Waals potentials $(Z=1)$ at arbitrary values of the parameter $\beta$ has been considered by some authors (see Alhassied 1987 and Ganesan and Taylor 1996). Many level crossings have been found, but the dynamics of these crossings are not clear.
In the present paper, we extend the previous works (Ganesan and Taylor 1996) to include not only the energy spectrum of the hydrogen atoms but also that of hydrogen-like atoms using the Hamiltonian defined by Eq. (2) in terms of the parameters $\beta$ and $D$ of the van der Waals potential. The Oscillator Representation Method (ORM) (Dineykhan and Efimov 1994) of the Schrödinger equation is employed for calculating the energy spectrum of the axially symmetric potentials. Moreover, the crossing behavior of the Rydberg states has been investigated.

The material of the paper is organized as follows: In section 2, the basic formulas of ORM are given. In section 3, the method is employed for calculating the energy spectrum of the generalized van der Waals Hamiltonian defined by Eq. (2). The energy spectrum results are studied and discussed for the hydrogen-like atoms in section 4.

\section{DERIVATION OF THE ORM FOR AXIALLY SYMMETRIC POTENTIALS}

In this paper, the ORM developed by Dineykhan and Efimov (1994) will be applied for calculating the energy spectrum of axially symmetric potentials. The first step in this direction is to change the variables in the original Schrödinger equation and obtain a modified equation, the solutions of which possess an oscillator behavior at large distances. Since this transformation is not canonical, it leads to a new system with different set of quantum numbers and wavefunctions, which contains, however, a subset of the original wavefunctions. The transformation of variables that leads to a Gaussian asymptotic behavior in the expanded space is one of the basic elements of the ORM. The next step is to write Hamiltonian in terms of the creation and annihilation operators $a+$ and $a$. The pure oscillator part with some unknown frequency! is extracted from the Hamiltonian, i.e. $H \rightarrow H_{0}+H_{I}=\omega a^{+} a+$ higher order terms. The remaining part, that is, the interaction Hamiltonian $H_{I}$ is represented in terms of normally ordered products of $a^{+}$and $a$. The requirement that this interaction Hamiltonian does not contain quadratic terms in canonical variables leads to the equation which determines the oscillator frequency $\omega$. This requirement is called the oscillator 
representation condition (ORC). Our starting point is to consider the Hamiltonian with an axially symmetric potential

$$
H=\frac{P^{2}}{2}+V(r, \cos \theta) \text {. }
$$

Axially symmetric potential has been used to study positron (electron) - diatomic molecules scattering (El-Aasser and Abdel-Raouf 2007). This kind of potentials were used for studying many quantum mechanical systems subjected to various perturbations such as Zeeman effect (Ruder et al 1994). They were also employed for investigating the behavior of quantum dots under magnetic fields (Dineykhan and Nazmitdimov 1997), and for calculating the energies and the eigenfunctions of deformed nuclei (Bohr and Mottelson 1975) and metallic clusters (de Heer 1993). In all these systems, Schrödinger equations could not be solved exactly and we are obliged to deal with approximations.

Our main goal in the present work is to study a quantum mechanical system governed by the axially symmetric potential defined at Eq. (3). The Schrödinger equation of this system can be written as

$$
\left\{-\frac{1}{2}\left[\frac{\partial^{2}}{\partial x^{2}}+\frac{\partial^{2}}{\partial y^{2}}+\frac{\partial^{2}}{\partial z^{2}}\right]+V(r, \cos \theta)\right\} \Psi(\vec{r})=E \Psi(\vec{r}),
$$

The wavefunction $\Psi(\vec{r})$ can be represented by

$$
\Psi(\vec{r})=\Phi_{v, m}(r) Y_{v, m}(\theta, \phi) .
$$

The Spherical Harmonics are defined by

$Y_{v, m}(\theta, \phi)=(-1)^{m} \frac{e^{i m \phi}}{\sqrt{2 \pi}} \sqrt{\frac{(2 v+1)}{2} \frac{\Gamma(v-m+1)}{\Gamma(v+m+1)}} P_{v}^{m}(\cos \theta)$.

$P_{\mathrm{v}}^{m}(\cos \theta)$ 's are the associated Legendre polynomials, $m=$ $0, \pm 1, \pm 2, \ldots$ are the azimuthal quantum numbers. $v$ is an arbitrary parameter which takes integer values only when the potential of the given system is spherically symmetric. Thus, the violation of the spherical symmetry property of the system is controlled by the parameter $v$, i.e. when $v$ assumes integer values, the functions $Y_{v, m}(\theta, \phi)$ are the spherical harmonics. The effects of the angular momentum operators $\hat{l}_{z}, l_{ \pm}$, and $\hat{l}^{2}$ on these functions are defined as

$$
\begin{aligned}
& \hat{l}_{z} Y_{v, m}=m Y_{v, m}, \hat{l}_{ \pm} Y_{v, m}=-\sqrt{v(v+1)-m(m \pm 1)} Y_{v, m \pm 1}, \\
& \hat{l}^{2} Y_{v, m}=v(v+1) Y_{v, m}
\end{aligned}
$$

Let us rewrite Schrödinger's equation (4) in the form

$$
\int d \vec{r} \Psi(\vec{r})\left[-\frac{1}{2} \Delta+V(r, \cos \theta)-E\right] \Psi(\vec{r})=0
$$

Substituting from (5) into (8) and taking (7) into account, we obtain

$$
\begin{aligned}
& \int_{0}^{\infty} d r\left(r \Phi_{v, m}(r)\right) \times \\
& \times\left[-\frac{1}{2}\left(\frac{d}{d r}\right)^{2}+\frac{v(v+1)}{2 r^{2}}+\left(\tilde{V}(r, v)-E_{m}\right)\right] \times \\
& \times\left(r \Phi_{v, m}(r)\right)=0 ;
\end{aligned}
$$

$$
\tilde{V}(r, v)=\frac{1}{N_{m}^{2}(\mathrm{v})} \int_{-1}^{1} d x P_{\mathrm{v}}^{m}(x) V(r, x) P_{\mathrm{v}}^{m}(x),
$$

$N_{m}(\mathrm{v})$ is a normalization constant determined by

$$
N_{m}^{2}(v)=\int_{-1}^{1} d x P_{v}^{m}(x) P_{v}^{m}(x)
$$

The first step in the ORM is to modify the variable $r$ in the radial equation (9) and the wavefunction $\Phi_{v, m}(r)$ in order to derive a modified Schrödinger equation having a solution with Gaussian asymptotic behavior. For this purpose we put, (see Dineykhan and Evmov 1994 for more details)

$$
r=q^{2 \rho} \quad \text { and } \quad \Phi_{v, m}(r)=q^{2 \rho v} \tilde{\Phi}_{v, m}\left(q^{2}\right)
$$

Substituting into equation (9) yields

$$
\begin{aligned}
& \int_{0}^{\infty} d q q^{d-1} \tilde{\Phi}_{v, m}\left(q^{2}\right)\left\{-\frac{1}{2}\left[\frac{\partial^{2}}{\partial q^{2}}+\frac{d-1}{q} \frac{\partial}{\partial q}\right]+\right. \\
& \left.+W\left(q, v, E_{m}\right)\right\} \tilde{\Phi}_{v, m}\left(q^{2}\right)=0
\end{aligned}
$$

where $d=2+2 \rho+4 \rho v$ and the potential $W(q, v, E m)$ is given by

$$
W\left(q, v, E_{m}\right)=4 \rho^{2} q^{4 \rho-2}\left[\tilde{V}(r, v)-E_{m}\right] .
$$

The first step towards the required solution is to consider the Schrödinger equation

$$
H \tilde{\Phi}_{v, m}\left(q^{2}\right)=\varepsilon(E) \tilde{\Phi}_{v, m}\left(q^{2}\right)
$$

such that

$$
\varepsilon(E)=0,
$$

which provides us with the energy spectrum $E$.

The second step is to represent the canonical variables (i.e. the coordinates and the momenta) in normal forms. For this purpose, the Hamiltonian $H$ should be written as

$$
H=\frac{1}{2}\left(P^{2}+\omega^{2} q^{2}\right)+\left(W(q)-\frac{1}{2} \omega^{2} q^{2}\right),
$$

where $\omega$ is the unknown oscillator frequency. In terms of the creation and annihilation operator's $a_{j}^{+}$and $a_{j}^{+}$, respectively, the $q$ 's and p's can be expressed by

$$
q_{j}=\frac{1}{\sqrt{2 \omega}}\left(a_{j}+a_{j}^{+}\right), \quad p_{j}=\frac{\sqrt{\omega}}{i \sqrt{2}}\left(a_{j}-a_{j}^{+}\right) .
$$

Therefore, the Hamiltonian given at equation (17) could be split to

$$
H=H_{0}+H_{I}+\varepsilon_{0}
$$

where

$$
\begin{gathered}
H_{0}=\omega\left(a^{+} a\right) \\
H_{I}=\int\left(\frac{d k}{2 \pi}\right)^{d} \tilde{W}_{d}\left(k^{2}\right) \exp \left(-\frac{k^{2}}{4 \omega}\right): e^{i k q}-1+\frac{k^{2} q^{2}}{2 d}:
\end{gathered}
$$


and

$$
\varepsilon_{0}=\frac{d \omega}{4} \int\left(\frac{d k}{2 \pi}\right)^{d} \tilde{W}_{d}\left(k^{2}\right) \exp \left(-\frac{k^{2}}{4 \omega}\right),
$$

where : * : is the symbol of normal ordering, $k q=\sum_{j} k_{j} q_{j}$. From the ORM, it follows that the frequency $\omega$ satisfies the equation

$$
\omega^{2}-\int\left(\frac{d k}{2 \pi}\right)^{d} \tilde{W}_{d}\left(k^{2}\right) \exp \left(-\frac{k^{2}}{4 \omega}\right) \frac{k^{2}}{d}=0 .
$$

Thus, the condition of the oscillator representation can be written as

$$
\frac{\partial}{\partial \omega} \varepsilon_{0}(E ; \omega, d)=0
$$

This equation determines the parameter $\omega=\omega(E, d)$ as a function of $E, d$ and other parameters included in the potential $\tilde{V}(r, v)$ defined at equation (10).

In the next section, the rules formulated above are used for calculating the energy spectrum of the generalized van der Waals potential.

\section{THE ENERGY SPECTRUM OF THE GENERALIZED VAN DER WAALS POTENTIAL}

The generalized van der Waals Hamiltonian given by (2) can be rewritten in the form

$$
\begin{aligned}
H & =\frac{1}{2} P^{2}-\frac{Z}{r}-\frac{(1-Z)^{2}}{4 D}+\frac{(1-Z)}{4 D^{2}} r \cos \theta-\gamma r^{2} \times \\
& \times\left[Z+\left(\beta^{2}(2-Z)-1\right) \cos ^{2} \theta\right]
\end{aligned}
$$

where $\gamma=1 / 16 D^{3}$. For this kind of potentials we put $\rho=1$ in the transformation (12).

Some of the physically meaningful and interesting situations of the Hamiltonian (24) (in the case $Z=1$ i.e. hydrogen atom) are as follows:

(i) For $\gamma=0$ and $\beta=0$, Eq. (24) represents the standard hydrogen atom problem (Goldstein 1980).

(ii) The case $\gamma \neq 0$ and $\beta=0$ corresponds to quadratic Zeeman problem (Kleppner 1991).

(iii) The case where $\gamma \neq 0$ and $\beta=1$ represents the so-called spherical quadratic Zeeman problem (Silva 1984) .

(iv) When $\gamma=1 / 16 D^{3}$ and $\beta=\sqrt{2}$, the system corresponds to the instantaneous van der Waals potential problem (Alhassid 1987).

After some simplifications, the modified Schrödinger equation takes the form

$$
\begin{aligned}
& \left\{-\frac{1}{2}\left[\frac{\partial^{2}}{\partial q^{2}}+\frac{d-1}{q} \frac{\partial}{\partial q}\right]-4 Z-4 E q^{2}-4 F q^{2}-4 G q^{6}\right\} \times \\
& \times \tilde{\Phi}_{v, m}\left(q^{2}\right)=0
\end{aligned}
$$

where $d=4+4 v ; F=\frac{1}{4 D}(1-Z)^{2}$;

$$
\begin{aligned}
G= & \gamma\left[Z+\left(\beta^{2}(2-Z)-1\right) A_{m}(v)\right] \\
& \text { and } A_{m}(v)=\frac{1}{N_{m}^{2}(v)} \int_{-1}^{1} d x\left[x P_{v}^{m}(x)\right]^{2}
\end{aligned}
$$

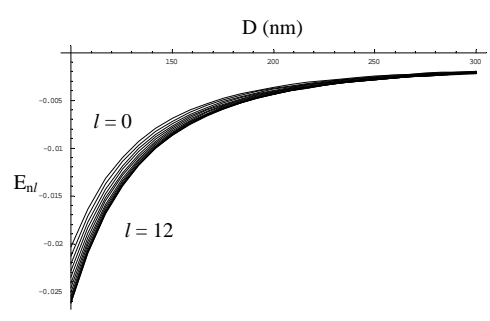

FIG. 1: The energy spectrum of the $n=20$ manifold of the hydrogen atom (see equation (30)) over atom-surface distance range from 100 $\mathrm{nm}$ to $300 \mathrm{~nm}$ at $\beta=0$.

Then after some calculations, the three components of the Hamiltonian presented at equation (18) take the forms

$$
\begin{gathered}
H_{0}=\omega\left(a^{+} a\right) \\
H_{I}=-\frac{6 G(d+4)}{\omega}: q^{4}:-4 G: q^{6}: \\
\varepsilon_{0}=\frac{\omega d}{4}-\frac{2 d E}{\omega}-\frac{2 d F}{\omega}-4 Z-\frac{G d(d+2)(d+4)}{2 \omega^{3}}
\end{gathered}
$$

Furthermore, the condition of the oscillator representation (23) can be written as

$$
\omega^{2}=-4(E+F)+\sqrt{16(E+F)^{2}-6 G(d+2)(d+4)}
$$

The term proportional to $\gamma$ could be considered as a minimal perturbation correction, so that $G<<1$. In this limit, or the zero approximation of OR, the parameter $v$ takes only integer values, i.e. $v=l$. The energy spectrum in the lowest approximation of the oscillator representation is determined by

$$
\varepsilon_{0}(E)+2 n_{r} \omega+\left\langle n_{r}\left|H_{I}\right| n_{r}\right\rangle=0 .
$$

The details of calculating the matrix elements $\left\langle n_{r}\left|H_{I}\right| n_{r}\right\rangle$ were presented previously by Dineykhan (1997).

Considering equations (27-29) provides us, after some analyses, with the energy spectrum

$$
\begin{aligned}
& E_{n l}=-\frac{Z^{2}}{2 n^{2}}-\frac{(1-Z)^{2}}{4 D}-\frac{G n^{2}}{2} \times \\
& \times\left[5 n^{2}-5(l+1)^{2}+(2 l+3)(l+2)\left(\frac{3}{Z^{2}}-2\right)\right]+O\left(\gamma^{2}\right)
\end{aligned}
$$

where $n=1+l+n r$ is the principle quantum number.

\section{DISCUSSION}

In this paper the effect of the generalized van der Waals potential on the Rydberg states of hydrogen-like atoms is investigated. The ORM technique is employed to solve the Schrödinger equation of the axially symmetric potentials analytically. The energy spectrum of the hydrogen-like atoms 


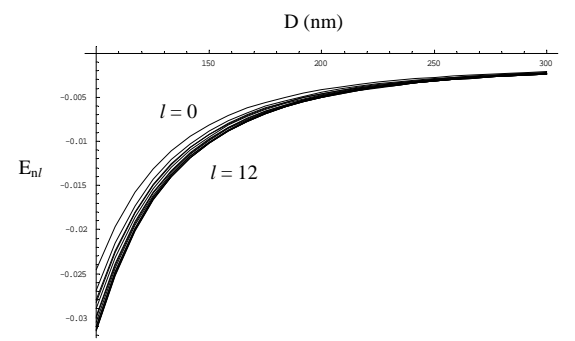

FIG. 2: The same as in Fig. 1, at $\beta=1$.

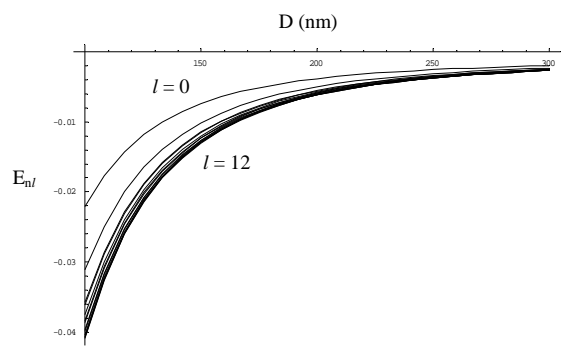

FIG. 3: The same as in Fig. 1, at $\beta=\sqrt{2}$.

are calculated analytically (see equation (30)). The results of the energy spectrum of the hydrogen-like atoms are represented in Figs. (1-11) for the Rydberg states $n=20$ of the hydrogen-like atoms over atom-surface distance $D$ ranging from 50 to $300 \mathrm{~nm}$ for some values of the orbital quantum number $l$ in the interval from 0 to 12 and arbitrary values of the parameter $\beta$ falling in the range $0 \leq \beta \leq 4$. In Figs. (1-3), the spectrum of the $n=20$ mainfold of the hydrogen atom $(Z=1)$ is plotted versus $\mathrm{D}$ for various values of the parameter $\beta$ for the states $l=0$ to $l=12$. It is clear that the states are crossing at some values of $\beta$ and some values of $D$ between 100 and $300 \mathrm{~nm}$. Out of these distances, we notice that there is a wide shift between the states. To clarify the crossing effect, which occurs between the states, we plotted in Figs. $(4,5)$ only the two states $l=1$ and $l=12$. and $l=2$ and $l=10$ respectively. The dynamics of these crossings can be interpreted as follows: From the expression (30), let $l_{0}$ be the fixed value of $l$, then by increasing $\beta$ we obtain the energy spectrum $E_{0}\left(\beta, l_{0}\right)$ of the state $l_{0}$. When $l=l_{0}+1$ we obtain

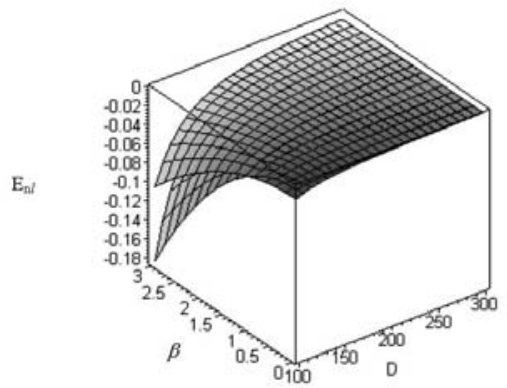

FIG. 4: The energy spectrum of the hydrogen atom for the levels $l=1$ and $l=12$, at $0 \leq \beta \leq 3$.

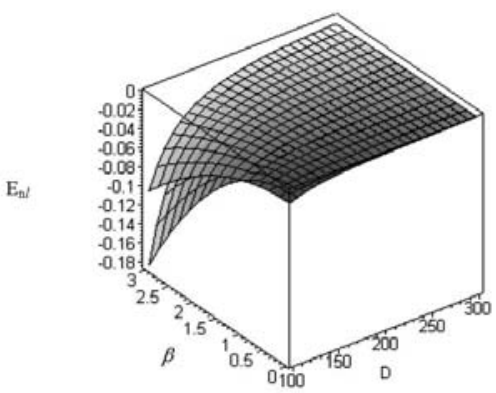

FIG. 5: The energy spectrum of the hydrogen atom for the levels $l=1$ and $l=12$, at $0 \leq \beta \leq 3$.

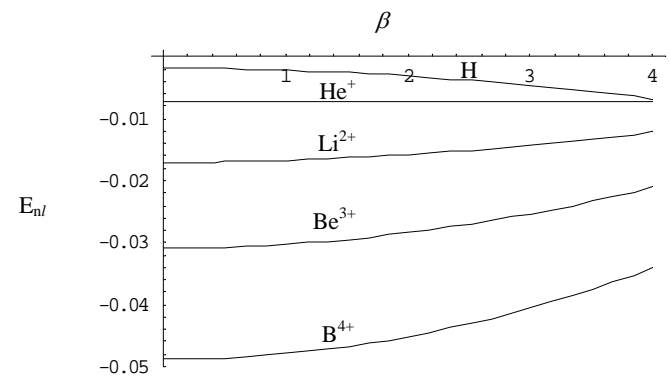

FIG. 6: The energy spectrum versus $\beta$ for $\mathrm{d}=300$ and $l=0$.

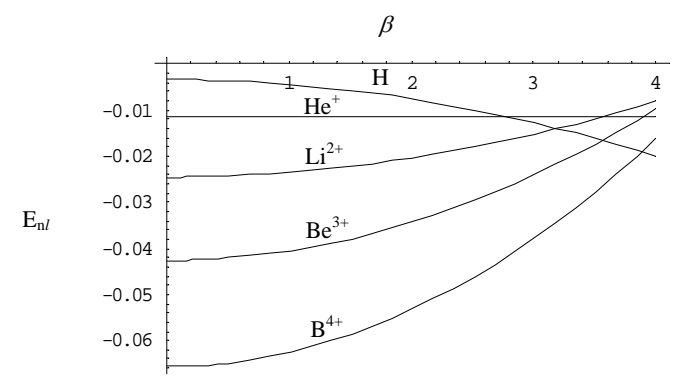

FIG. 7: The energy spectrum versus $\beta$ for $d=200$ and $l=0$.

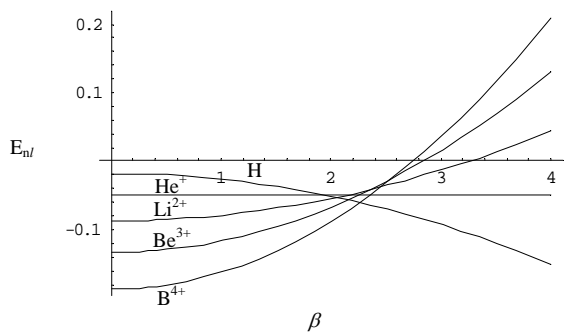

FIG. 8: The energy spectrum versus $\beta$ for $d=100$ and $l=0$. 


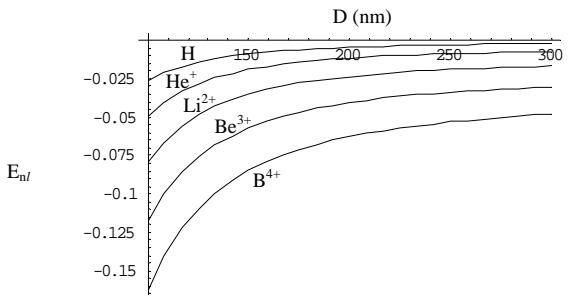

FIG. 9: The energy spectrum versus $\mathrm{D}$ for $\beta=1$ and $l=0$.

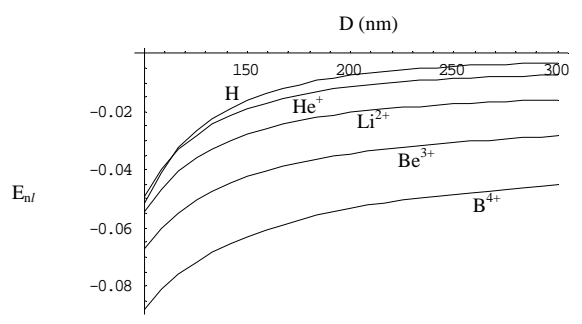

FIG. 10: The energy spectrum versus $\mathrm{D}$ for $\beta=2$ and $l=0$.

the energy spectrum $E_{1}\left(\beta, l_{0}+1\right)$ of the state $l_{0}+1$ and on increasing $\beta ; E_{1}\left(\beta, l_{0}+1\right)$ increases but with rate less than the rate of increasing $E_{0}\left(\beta, l_{0}\right)$. Therefore the two states are crossing at a definite value of $\beta=\beta_{0}$. On increasing further the value of $l$, e.g. to $l=l_{0}+2$, we obtain a crossing between the two states $E_{2}\left(\beta, l_{0}+2\right)$ and $E_{0}\left(\beta, l_{0}\right)$ at some values of $\beta=\beta_{1}$, and also between the states $E_{2}\left(\beta, l_{0}+2\right)$ and $E_{1}\left(\beta, l_{0}+1\right)$ there is a crossing at some values of $\beta=\beta_{2}$. For the other values of $l$, the crossing between every two states occurs at other values of $\beta$. Consequently, at fixed value of $\beta$ in the $E D$ plane, there is only one crossing between all the states. This result agrees quite well with the results obtained by other authors. The energy does not change significantly with the variation of $\beta$ in figures (6) and (7) until $\beta$ exceeds 2.5 where we get level crossings between the atoms $\mathrm{H}, \mathrm{He}^{+}$, $L i^{2}+, B e^{3+}, B^{4+}$ for $l=0$. We may notice that with decreasing the value of $D$ the energies become more negative i.e. the atoms are more bounded due to a stronger attractive potential. In Fig. (8), the energies remains constant until $\beta$ exceeds 2 where we get level crossings after which we may get unbounded atoms where the repulsive part of the potential exceeded the attractive one (see Eq. (2)). Figs $(6,7,8)$ show that $\mathrm{He}$ ion does not change with $\beta$ because it is one of the noble gases, the most stable atomic systems. In Figs. (9) and (10), for constant values of $\beta$, as D increases the energies also

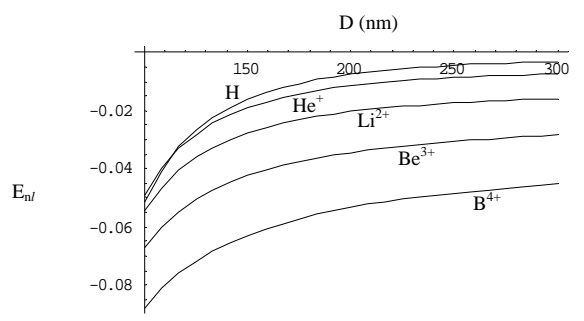

FIG. 11: The energy spectrum versus $D$ for $\beta=3$ and $l=0$.

increase and saturate. The atoms get less bounded due to the less attractive potential. In Fig. (11), at small values of D we may get unbounded atoms at $\beta=3$ however by increasing $\mathrm{D}$ all atoms get bounded due to the balance between the two parts of the potential where the attractive one supersedes.
[1] Alhassied Y, Hinds E A, and Meschede D 1987, Phys.Rev.Lett. 591545.

[2] Bohar A, and Mottelson B R 1975, Nuclear Structure, vol 2 (New York: Benjamin).

[3] de Heer W A 1993, Mod.Phys. 65 611; Barck M 1993, Rev. Mod. Phys. 65677.

[4] Dineykhan M and Efimov G V 1994, Few-Body Systems 16 59 ;

[5] Dineykhan M and Nazmitdinov R G 1997, Phys.Rev.B 2020.

[6] Dineykhan M 1997, Z.Phys.D 4177.

[7] El-Aasser M A and Abdel-Raouf M A 2007, J. Phys. B: At. Mol. Opt. Phys. 401801.
[8] Goldstein H 1980, classical mechanics (London: AddisonWesly).

[9] Ganesan K and Taylor K T 1996, J.Phys.B: At.Mol.Opt.Phys. 291293.

[10] Kleppner D 1991, Phys.Today 44 9; Friedrich H 1992, Phys. World 5 (4) 32.

[11] Ruder H, Wunner G, Herold H, and Geyer F 1994, Atoms in Strong Magnetic Fields (Berlin: Springer).

[12] Series G W, 1988, The Spectrum of Atomic Hydrogen Advances (Singapore: World Scientific).

[13] Silva J R and Canuto S 1984, Phys. Lett. 101A 326. 